\title{
Non Equilibrium Dissociation of HF in Electrical Discharges: The Role of Vibrational Excitation*
}

\author{
M. Capitelli and M. Dilonardo \\ Centro di Studio per la Chimica dei Plasmi del C.N.R. \\ Dipartimento di Chimica dell'Università di BARI \\ Z. Naturforsch. 33a, 1085-1089 (1978); received July 3, 1978
}

Dissociation rates of $\mathrm{HF}$ in electrical discharges have been calculated by solving a system of vibrational master equations, including e-V (electron-vibration), V-V (vibration-vibration) and V-T (vibration-translation) energy exchanges. The results show that large dissociation rates can be obtained by this mechanism only when the e-V pumping rates overcome the V-T deactivating rates. This occurs for electron number densities larger than $10^{13} \mathrm{~cm}^{-3}$.

Finally the close connection of the present results with the corresponding ones obtained by using as vibrational excitation source an IR powerfull laser is shown and discussed.

\section{Introduction}

We have recently proposed a vibrational model for explaining the dissociation rates of homonuclear diatomic species in electrical discharges [1]. According to this model the electrons of the discharge populate the first vibrational levels of the ground electronic state of the molecule, thus introducing a vibrational disequilibrium with respect to the gas temperature.

The vibrational quanta are then transported up to the dissociation limit by means of V-V (vibration-vibration) energy exchanges. The V-T (vibration-translation) processes on the contrary tend to destroy this mechanism, by trying to restore a Boltzmann vibrational population at the gas temperature $\left(T_{\mathrm{g}}\right)$.

This mechanism can be very effective for those molecules which possess large e- $\mathrm{V}$ (electronvibration) rates combined with small V-T deactivating ones.

In this paper we extend this model to the $\mathrm{HF}$ molecule and the results will be compared with the values recently obtained by Kompa et al. [2], who studied the HF dissociation induced by IR pulsed laser.

\section{Method of Calculation}

According to the vibrational mechanism outlined in Refs. [1, 2], the dissociation rate $\left(\mathrm{cm}^{-3} \mathrm{~s}^{-1}\right)$ of

\footnotetext{
* Work presented to the "National Meeting on Quantum Electronics and Plasmas", Frascati (Roma) 17-19 May 1978.
}

Reprint requests to Dr. Capitelli, Dipartimento di Chimica Università - Via Amendola 173 - I-70126 Bari (Italy). the $\mathrm{HF}$ in electrical discharges can be written as

$$
\begin{aligned}
v_{\mathrm{D}}= & \frac{\partial N_{v^{\prime}+1}}{\partial t}=\sum_{i=1}^{3} N_{i} N_{v^{\prime}} K_{v^{\prime}, v^{\prime}+1}^{i} \\
& +N_{v^{\prime}} \sum_{v=1}^{v^{\prime}} N_{v} K_{v, v-1}^{v^{\prime}, v^{\prime}+1}+n_{e} \sum_{v=1}^{v^{\prime}} N_{v} K_{v, v^{\prime}+1}^{e}
\end{aligned}
$$

where $N_{v}$ is the population density of $v$ th level $\left(v^{\prime}=19\right.$ is the last bound vibrational level of the ground electronic state of $\mathrm{HF}$ and $v^{\prime}+1$ is the dissociation level $[1,2])$ and $i=1,2,3$ corresponds respectively to $\mathrm{HF}, \mathrm{H}, \mathrm{F}$.

The rate coefficients appearing in Eq. (1) refer to the following processes

$$
\begin{aligned}
\mathrm{HF}\left(v^{\prime}\right) & +\mathrm{HF}(\mathrm{H}, \mathrm{F}) \stackrel{K_{v^{\prime}, v^{\prime}+1}^{\mathrm{HF}, \mathrm{H}} \rightarrow \mathrm{HF}\left(v^{\prime}+1\right)}{ } \\
& +\mathrm{HF}(\mathrm{H}, \mathrm{F}) \rightarrow \mathrm{H}+\mathrm{F}+\mathrm{HF}(\mathrm{H}, \mathrm{F}), \\
\mathrm{HF}\left(v^{\prime}\right) & +\mathrm{HF}(v) \stackrel{K_{v, v-1}^{v^{\prime}, v^{\prime}+1}}{\rightarrow} \mathrm{HF}\left(v^{\prime}+1\right) \\
& +\mathrm{HF}(v-1) \rightarrow \mathrm{H}+\mathrm{F}+\mathrm{HF}(v-1), \\
\mathrm{HF}(v) & +\mathrm{e} \stackrel{K_{v, v^{\prime}+1}^{e}}{\rightarrow} \mathrm{HF}\left(v^{\prime}+1\right) \\
& +\mathrm{e} \rightarrow \mathrm{H}+\mathrm{F}+\mathrm{e} .
\end{aligned}
$$

It should be noted that we are considering only the dissociation rate of the pure vibrational mechanism, i.e. contributions coming from the direct electronic mechanism

$$
\mathrm{e}+\mathrm{HF} \rightarrow \mathrm{e}+\mathrm{HF}^{*} \rightarrow \mathrm{e}+\mathrm{H}+\mathrm{F}
$$

and from the dissociative attachment

$$
\mathrm{e}+\mathrm{HF} \rightarrow \mathrm{H}+\mathrm{F}^{-}
$$

are not considered in the present paper.

Equation (1) is then coupled to a system of $v^{\prime}$ vibrational master equations [1], which describe 
the temporal evolution of the vibrational levels under the action of e-V, V-V and V-T energy exchanges.

A typical equation of this system $[1,2]$ is written as

$$
\begin{aligned}
\frac{\partial N_{v}}{\partial t}= & \sum_{i=1}^{3} N_{i}\left[N_{v-1} K_{v-1, v}^{i}+N_{v+1} K_{v+1, v}^{i}\right. \\
& \left.-N_{v}\left(K_{v, v-1}^{i}+K_{v, v+1}^{i}\right]\right] \\
& +\sum_{w=0}^{v^{\prime}} N_{w}\left[N_{v-1} K_{w, w-1}^{v-1, v}+N_{v+1} K_{v+1, v}^{w, w+1}\right. \\
& \left.\quad-N_{v}\left(K_{v, v-1}^{w, w+1}+K_{w, w-1}^{v, v+1}\right)\right] \\
& +\sum_{w=0}^{v^{\prime}} n_{e}\left[N_{w} K_{w, v}^{e}-N_{v} K_{v, w}^{e}\right] \\
& +N_{v+1} A_{v+1, v}-N_{v} A_{v, v-1}
\end{aligned}
$$

$\left(A_{v, v-1}\right.$ is the spontaneous emission coefficient).

It should be noted that Eqs. (1), (7) disregard the recombination process, taking into account the deactivation of vibrational energy by the atoms $(\mathrm{H}, \mathrm{F})$. As for the rate coefficients appearing in Eqs. (1), (7), they have been calculated as follows.

1) The e-V rates

$\mathrm{e}+\mathrm{HF}(v=0) \rightarrow \mathrm{HF}^{-} \stackrel{K_{0 v}^{e}}{\longrightarrow} \mathrm{e}+\mathrm{HF}(v=1,2)$

have been obtained by numerical integration of the experimental cross section of Rohr and Linder [3] over a Maxwell electron distribution function. This last hypothesis does not affect too much the e-V rates, because the thresholds of these processes overlap with the bulk of electron distribution function, which in turn is not affected by inelastic processes [4].

The missing e- $\mathrm{V}$ rates have been simply obtained by imposing $K_{01}^{e}=K_{1,2}^{e}=K_{2,3}^{e}$ and so on $\left(K_{0 v}^{e}=0\right.$, $v>2$ ), while the detailed balance has been used for the $\mathrm{e}-\mathrm{V}$ reverse rates.

2) V-T and V-V rates have been taken from the work of Kompa et al. [2], even though other rates have appeared in the literature since this work [5]. However their incorporation in our work is not done for a consistent comparison of dissociation rates induced by electrons and by IR laser.

The system of $v^{\prime}+1$ differential equations has been numerically integrated with the following initial condition

$$
\begin{aligned}
& N_{v}(t=0)=0 \quad \text { for } \quad v \neq 0, \\
& N_{v}(t=0)=N_{\mathrm{HF}}^{0}, \quad v=0, \\
& N_{\mathrm{H}}=N_{\mathrm{F}}=0
\end{aligned}
$$

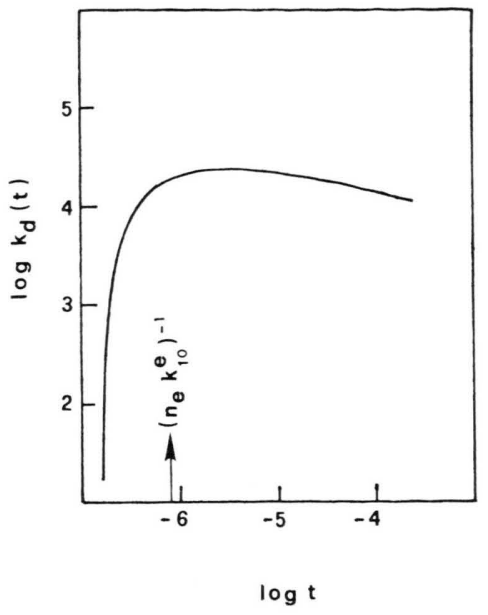

Fig. 1. Dissociation constant $\left(\mathrm{s}^{-1}\right)$ as a function of time $\left(n_{e}=10^{14} \mathrm{~cm}^{-3}, T_{\mathrm{g}}=300 \mathrm{~K}, p=3\right.$ torr, $\left.T_{e}=10000 \mathrm{~K}\right)$.

at different gas temperatures $\left(300 \mathrm{~K} \leqq T_{\mathrm{g}} \leqq 1000 \mathrm{~K}\right)$, pressures $(3 \leqq p \leqq 60$ torr $)$, electron densities $\left(10^{11} \leqq\right.$ $\left.n_{e} \leqq 10^{14} \mathrm{~cm}^{-3}\right)$. In all calculations the electron temperature has been considered equal to $T_{e}=$ $10000 \mathrm{~K}$, which gives very high e-V rates minimizing the dissociation processes coming through other channels.

After a transient period, which is approximately given by $\left(n_{e} K_{01}^{e}\right)^{-1}$, the pseudofirst order constant $k_{d}(t)=v_{\mathrm{D}} / \sum_{0}^{v^{\prime}} N_{v}$ settles to a quasistationary value $k_{d} s$ [1], as can be appreciated in Figure 1. This value can be taken as an indication of the effectiveness of the vibrational mechanism in dissociating HF.

\section{Results}

$\mathrm{HF}$ is an interesting system from the point of view of the vibrational kinetics, presenting infact large e- $V$ rates $\left(K_{01}^{e} \sim 10^{-8} \mathrm{~cm}^{3} \mathrm{~s}^{-1}\right.$ at $\left.T_{e}=10000 \mathrm{~K}\right)$ combined, unfortunately, with high deactivation rates of the process

$\mathrm{HF}(v=1)+\mathrm{HF} \stackrel{K_{10}^{\mathrm{HF}}}{\longrightarrow} \mathrm{HF}(v=0)+\mathrm{HF}$.

In our previous works [1] (see also Ref. [6]) we have shown that the efficiency of the vibrational mechanism in dissociating diatomic species is strictly related to the possibility of creating a large "vibrational" temperature $\Theta_{1}=E_{10} / \ln \left(N_{0} / N_{1}\right)\left(E_{10}\right.$ is the energy of the transition $0-1$ and $N_{0}, N_{1}$ denote in this order the population densities of the 
ground and of the first vibrational level. Large $\Theta_{1}$ values can be obtained when the pumping rate $\left(n_{e} K_{01}^{e}\right)$ overcomes the most important V-T deactivation process (in this case $N_{\mathrm{HF}} K_{1,0}^{\mathrm{HF}}$ ). To obtain large $\Theta_{1}$ values, therefore, one must consider $\mathrm{HF}$ electrical discharges with electron densities $n_{e}\left(\mathrm{~cm}^{-3}\right)$ obeying the inequality

$$
n_{e}>\frac{K_{10}^{\mathrm{HF}}}{K_{01}^{e}} N_{\mathrm{HF}} .
$$

Values of $\left(K_{10}^{\mathrm{HF}} / K_{01}^{e}\right) N_{\mathrm{HF}}$ can be calculated at different $T_{\mathrm{g}}$ and pressures. One can note that appreciable "vibrational temperatures" can be only obtained at high $n_{e}$.

As an example, at $T_{\mathrm{g}}=300 \mathrm{~K}, p=3$ torr one must have at least $n_{e}=2.4 \cdot 10^{13} \mathrm{~cm}^{-3}$ to hope for the vibrational kinetics, while at the same gas temperature and pressure $=60$ torr, we must exceed $n_{e}=4.7 \cdot 10^{14} \mathrm{~cm}^{-3}$. The numerical results confirm these considerations.

Figure 2, infact, shows the behaviour of $k_{d}{ }^{s}$ as a function of pressure $\left(n_{e}=10^{14} \mathrm{~cm}^{-3}, T_{e}=10000 \mathrm{~K}\right.$, $T_{\mathrm{g}}=300 \mathrm{~K}$ ). One can note that the quasistationary $k_{d} s$ values decrease from approximately $10^{4} \mathrm{~s}^{-1}$ to $10^{3} \mathrm{~s}^{-1}$ by increasing the pressure from $p=3$ to

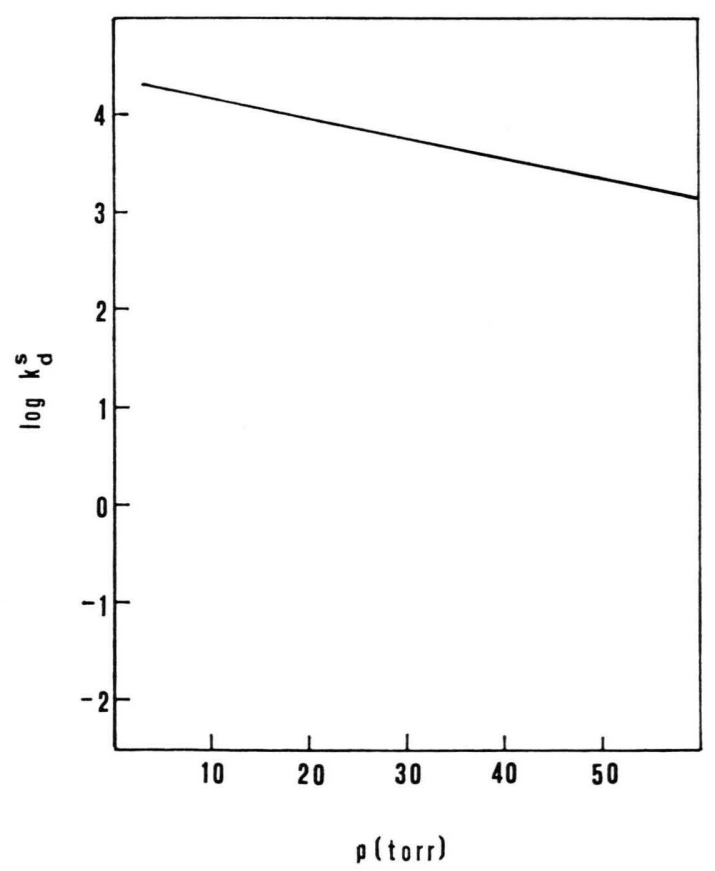

Fig. 2. Values of $k_{d}^{s}\left(\mathrm{~s}^{-1}\right)$ as a function of pressure (same conditions of Fig. 1).

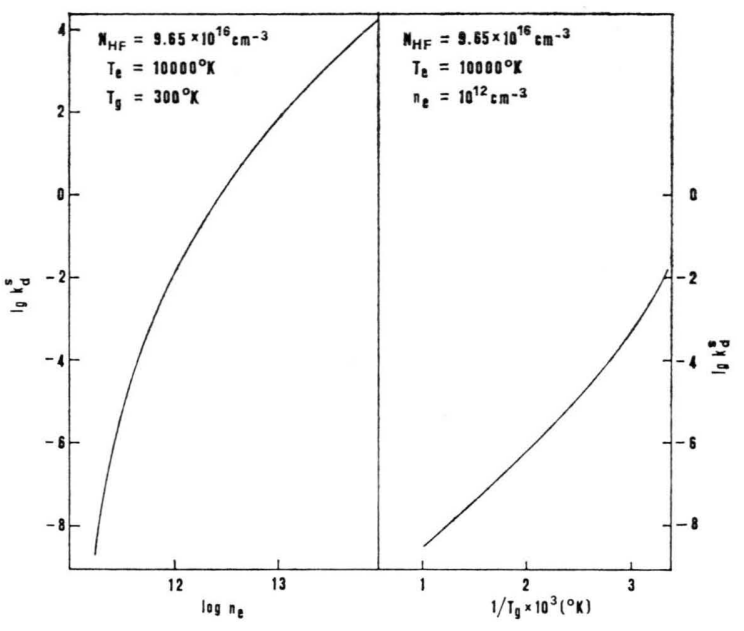

Fig. 3. a) Values of $k_{d}^{s}$ as a function of $n_{e}\left(\mathrm{~cm}^{-3}\right)(p=3$ torr, $T_{\mathrm{g}}=300 \mathrm{~K}$ );

b) Values of $k_{d}^{s}$ as a function of $1 / T_{\mathrm{g}}\left(N_{\mathrm{HF}}^{0} \cong 10^{17} \mathrm{~cm}^{-3}\right.$, $\left.n_{e}=10^{12} \mathrm{~cm}^{-3}\right)$.

60 torr, while the vibrational temperatures decrease from $4200 \mathrm{~K}$ to $2500 \mathrm{~K}$.

Figure 3 a shows the behaviour of $k_{d} s$ as a function of $n_{e}$, while Fig. $3 \mathrm{~b}$ reports $k_{d} s$ values as a function of $1 / T_{\mathrm{g}}$.

The trend of $k_{d} s$ versus $n_{e}$ can be understood on the qualitative considerations previously reported.

As for the dramatic decrease of $k_{d} s$ with increas ing $T_{\mathrm{g}}$, this is in line with our previous work [1]. It can be understood by recalling that the increase of $T_{\mathrm{g}}$ increases the reverse $\mathrm{V} \cdot \mathrm{V}$ processes, thereby destroying the $\mathrm{V} \cdot \mathrm{V}$ up mechanism discussed in References [1-2]. Figure 4 shows typical $N_{v}$ distributions at different times for the conditions which, in the present work, yielded the largest $k_{d} s$ values. In the same figure we have also reported the atom concentration $\left(\mathrm{cm}^{-3}\right)$ as a function of time. It should be noted that the $N_{v}$ distribution does not presents a Boltzmann tail for the conditions of Figure 4. This means that the $\mathrm{V}-\mathrm{V}$ processes dominate the V-T ones, due to the large vibrational temperature obtained at $n_{e}=10^{14} \mathrm{~cm}^{-3}, T_{\mathrm{g}}=$ $300 \mathrm{~K}$ and $p=3$ torr. As for the atom production, we can see (Fig. 4) that our mechanism without recombination is able to dissociate all $\mathrm{HF}$ molecules in a time of the order of $10^{-4} \mathrm{~s}$. It is worth noting that the atom concentration reaches a value of $2.10^{15} \mathrm{~cm}^{-3}$ at $t=10^{-6} \mathrm{~s}$, which is the characteristic time for pulsed discharges with $n_{e}=10^{14} \mathrm{~cm}^{-3}$ (see for example Ref. [7]). 


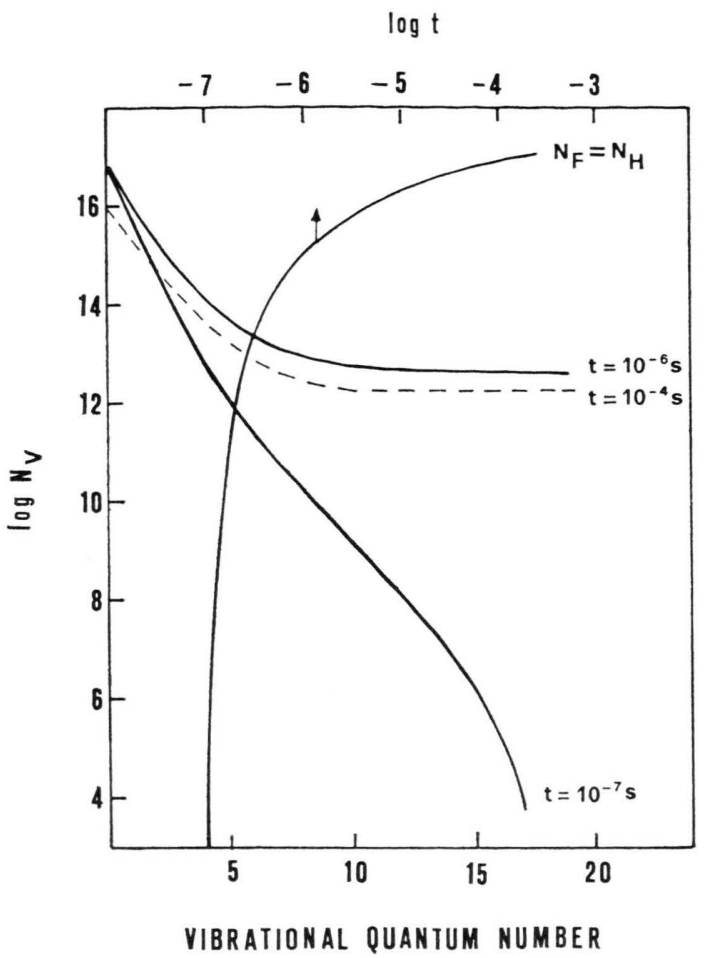

Fig. 4. Vibrational distribution $N_{v}\left(\mathrm{~cm}^{-3}\right)$ as a function of the vibrational quantum number at different times and atom concentration $\left(\mathrm{cm}^{-3}\right)$ as a function of time (s) (same conditions of Figure 1).

A balance between the vibrational quanta introduced by e- $V$ energy exchanges and the corresponding quanta lost by decay channels can be approximately written as follows [6]

$$
\begin{aligned}
n_{e} N_{v=0} K_{01}^{e} \cong & n_{e} N_{1} K_{10}^{e} \\
& +\sum_{i=\mathrm{HF}, \mathrm{H}, \mathrm{F}} N_{i} \sum_{v=1}^{v^{\prime}} N_{v} K_{v, v-1}^{i} \\
& +N_{\mathrm{HF}} k_{d^{s}} D / E_{10},
\end{aligned}
$$

where the three terms at the right of Eq. (12) represent in that order the vibrational quanta lost by superelastic collisions (the reverse of process (8)), $\mathrm{V}-\mathrm{T}$ deactivation and dissociation.

This last term destroys $D / E_{10}$ quanta ( $D$ is the dissociation energy) for any reactive collision [6]. It should be noted that $\mathrm{V}-\mathrm{V}$ terms do not appear in Eq. (12), since V-V collisions conserve vibrational quanta. Equation (12) applied to the $N_{v}$ distribution of Fig. 4 shows that all three decay channels of Eq. (12) are important in destroying vibrational quanta.

\section{Comparison with the IR Laser Induced Process}

The results reported in the present paper are very similar to those discussed by Kompa et al. [2], who studied the same mechanism induced by IR laser. These authors considered also the rotational structure of the first vibrational levels $(v \leqq 4)$ of $\mathrm{HF}$ due to the coupling of these levels with the laser input.

To a first approximation we neglect the rotational structure and we consider the pumping rate $0-1$ induced by the IR pulsed HF laser (duration $400 \mathrm{~ns})$ used in Reference [2].

At the laser power $W=27.10^{6} \mathrm{~W} / \mathrm{cm}^{2}$, we obtain a pumping rate

$$
\begin{aligned}
& P_{01} \sim \sigma_{01} W_{01} / E_{10} \sim 3 \cdot 10^{9} \mathrm{~s}^{-1} \\
& \left(\sigma_{01} \sim 10^{-16} \mathrm{~cm}^{2}, W_{01} \sim 10^{\circ} \mathrm{W},\right. \\
& \left.E_{10}=7.86 \cdot 10^{-20} \text { joules }\right) .
\end{aligned}
$$

This large pumping rate is able to overcome the vibrational deaxcitation of process (10), thereby introducing vibrational quanta in the $\mathrm{HF}$ system. $\mathrm{V}-\mathrm{V}$ processes will transport these quanta up to the dissociation limit.

The vibrational distributions obtained in Ref. [2] are very similar to those reported in the present work, while the dissociation constant calculated by Kompa et al. $\left(p_{\mathrm{HF}}=60\right.$ torr, $\left.W=27 \cdot 10^{6} \mathrm{~W} / \mathrm{cm}^{2}\right)$ is several orders of magnitude larger than our $k_{d} s$ value obtained at the same pressure with $n_{e}=10^{14} \mathrm{~cm}^{-3}\left(T_{\mathrm{g}}=300 \mathrm{~K}\right)$. This is due to the fact that the IR pumping rates used in Ref. [2] strongly exceed our e-V rates $\left(n_{e} K_{01}^{e} \sim 10^{6} \mathrm{~s}^{-1}\right.$ at $\left.n_{e}=10^{14} \mathrm{~cm}^{-3}, T_{e}=10000 \mathrm{~K}\right)$.

\section{Conclusions}

It should be interesting as a conclusion to predict how the present results can be extended to other similar systems.

Let us consider an $\mathrm{HCl}$ electrical discharge. In this case the e- $\mathrm{V}$ pumping rate of the process

$$
\mathrm{e}+\operatorname{HCl}(v=0) \rightarrow \mathrm{e}+\operatorname{HCl}(v=1)
$$

has the same order of magnitude as the process (8), while the deactivation rate of the process

$$
\mathrm{HCl}(v=1)+\mathrm{HCl} \rightarrow \mathrm{HCl}(v=0)+\mathrm{HCl}
$$

is several orders of magnitude smaller than process (10). 
Apparently, therefore, one should expect that the production of $\mathrm{H}, \mathrm{Cl}$ atoms by the pure vibrational mechanism could occur at electron number densities lower than those necessary for $\mathrm{HF}$ [8].

However, small quantities of $\mathrm{H}$ and $\mathrm{Cl}$ atoms strongly deactivate the vibrational content of $\mathrm{HCl}$ through the process [9]

$\mathrm{HCl}(v=1)+\mathrm{H}(\mathrm{Cl}) \rightarrow \mathrm{HCl}(v=0)+\mathrm{H}(\mathrm{Cl})$,

selflimiting the atom production by the pure vibrational mechanism.

On the other hand, if the atoms come through other channels (direct electronic mechanism, dissociative attachment) the vibrational content of

[1] M. Capitelli, M. Dilonardo, and E. Molinari, Chem. Phys. 20, 417 (1977); M. Capitelli and M. Dilonardo, Chem. Phys. 24, 417 (1977); 30, 95 (1978); Rev. de Phys. Appliquée 13, 115 (1978).

[2] H. Pummer, D. Proch, U. Schmailzl, and K. L. Kompa, J. Phys. D.; Appl. Phys. 11, 101 (1978); U. Schmailzl, H. Pummer, D. Proch, and K. L. Kompa, J. Phys. D.; Appl. Phys. 11, 111 (1978).

[3] K. Rohr and F. Linder, J. Phys. B, 9, 2521 (1976).

[4] M. Capitelli and M. Dilonardo, to be published.
$\mathrm{HCl}$ will be completely destroyed by process (16) which has a very large deactivation rate.

This means that, once again, the dissociation of $\mathrm{HCl}$ through a vibrational mechanism will occur only at very high electron densities, similar to those discussed for HF.

\section{Acknowledgements}

This work has been partially supported by Ministry of Education through the special law 286 (77-78).

We thank Mr. U. Farella for the drawings and Mr. G. Indelli for typewriting the manuscript.

[5] see for ex. R. L. Wilkins, J. Chem. Phys. 67, 5838 (1977).

[6] B. F. Gordiets, S. S. Mamedov, and L. Shelepin, Sov. Phys. JEPT 40, 640 (1975).

[7] S. D. Rockwood, J. E. Brau, W. A. Proctor, and G. H. Canavan, IEEE J. Quantum Electron. QE 9, 120 (1973).

[8] R. Taylor, G. Caledonia, P. Lewis, P. Wu, J. D. Teare, and J. Cronin,"Analytic modeling of electrically excited $\mathrm{D}_{2} / \mathrm{HCl}$ laser experiments" PSl Tr-58 (1976); see also AIAA J. 15, 1339 (1977).

[9] R. L. Wilkins, J. Chem. Phys. 63, 534 (1975). 